\title{
Clinical Characteristics and Prognosis of Coexisting Thyroid Cancer in Patients with Graves' Disease: A Retrospective Multicenter Study
}

\author{
Jee Hee Yoon ${ }^{1}$, Meihua Jin², Mijin Kim³ , A Ram Hong ${ }^{1}$, Hee Kyung Kim , Bo Hyun Kim³ ${ }^{3}$ Won Bae Kim², \\ Young Kee Shong ${ }^{2}$, Min Ji Jeon ${ }^{2}$, Ho-Cheol Kang ${ }^{1}$
}

${ }^{1}$ Division of Endocrinology and Metabolism, Department of Internal Medicine, Chonnam National University Medical School, Gwangju; ${ }^{2}$ Division of Endocrinology and Metabolism, Department of Internal Medicine, Asan Medical Center, University of Ulsan College of Medicine, Seoul; ${ }^{3}$ Division of Endocrinology and Metabolism, Department of Internal Medicine, Biomedical Research Institute, Pusan National University Hospital, Busan, Korea

Background: The association between Graves' disease (GD) and co-existing thyroid cancer is still controversial and most of the previously reported data have been based on surgically treated GD patients. This study investigated the clinicopathological findings and prognosis of concomitant thyroid cancer in GD patients in the era of widespread application of ultrasonography.

Methods: Data of GD patients who underwent thyroidectomy for thyroid cancer between 2010 and 2019 in three tertiary hospitals in South Korea (Asan Medical Center, Chonnam National University Hwasun Hospital, and Pusan National University Hospital) were collected and analyzed retrospectively. In the subgroup analysis, aggressiveness and clinical outcomes of thyroid cancer were compared nodular GD and non-nodular GD groups according to the presence or absence of the thyroid nodules other than thyroid cancer (index nodules).

Results: Of the 15,159 GD patients treated at the hospitals during the study period, 262 (1.7\%) underwent thyroidectomy for coexisting thyroid cancer. Eleven patients (4.2\%) were diagnosed with occult thyroid cancer and 182 patients (69.5\%) had microcarcinomas. No differences in thyroid cancer aggressiveness, ultrasonographic findings, or prognosis were observed between the nodular GD and non-nodular GD groups except the cancer subtype. In the multivariate analysis, only lymph node (LN) metastasis was an independent prognostic factor for recurrent/persistent disease of thyroid cancer arising in GD $(P=0.020)$.

Conclusion: The prevalence of concomitant thyroid cancer in GD patients was considerably lower than in previous reports. The clinical outcomes of thyroid cancer in GD patients were also excellent but, more cautious follow-up is necessary for patients with LN metastasis in the same way as for thyroid cancer in non-GD patients.

Keywords: Graves disease; Thyroid neoplasms; Prevalence; Aggression; Prognosis

Received: 4 August 2021, Revised: 5 October 2021, Accepted: 20 October 2021

Corresponding authors: Ho-Cheol Kang

Division of Endocrinology and Metabolism, Department of Internal Medicine, Chonnam National University Medical School, 160 Baekseo-ro, Dong-gu, Gwangju 61469, Korea

Tel: +82-61-379-7620, Fax: +82-61-379-7628, E-mail: drkang@chonnam.ac.kr

Min Ji Jeon

Division of Endocrinology and Metabolism, Department of Internal Medicine, Asan Medical Center, University of Ulsan College of Medicine, 88 Olympic-ro 43-gil, Songpa-gu, Seoul 05505, Korea

Tel: +82-2-3010-1317, Fax: +82-2-3010-6962, E-mail: mj080332@gmail.com

\section{Copyright $(2021$ Korean Endocrine Society}

This is an Open Access article distributed under the terms of the Creative Commons Attribution Non-Commercial License (https://creativecommons.org/ licenses/by-nc/4.0/) which permits unrestricted non-commercial use, distribution, and reproduction in any medium, provided the original work is properly cited. 


\section{INTRODUCTION}

Graves' disease (GD) is an autoimmune thyroid disease (AITD) that most frequently causes hyperthyroidism [1]. Binding to the G-protein couple thyrotropin receptor of circulating anti- thyroid-stimulating hormone (TSH) receptor antibodies results in thyrotoxicosis due to excessive thyroid hormone synthesis and thyroid gland hypertrophy $[2,3]$. The prevalence of thyroid cancer in GD patients varies widely, but a recent meta-analysis found a roughly 2.5-fold higher rate of thyroid cancer in GD patients than in the general population [4]. Early findings suggested that GD has a protective effect against thyroid cancer [5-7], but recent studies have revealed a higher incidence of thyroid cancer in patients with GD, particularly surgically treated patients $[8,9]$. Two nationwide cohort studies in Taiwan and Sweden evaluated the association between thyroid cancer and GD, and both found a higher incidence of thyroid cancer during the early GD follow-up period [10,11]. Routinely performed ultrasonography (US) in GD patients during disease surveillance might be related to overdiagnosis of thyroid cancer. Use of screening US for thyroid disease increases the probability of detecting thyroid nodules and patients with thyroid nodules may be at an increased risk for thyroid cancer [12]. A review and meta-analysis of surgically treated GD patients revealed that thyroid nodules are a risk factor of thyroid cancer [13], but the real incidence of thyroid cancer in GD patients who are treated with or without surgery is uncertain.

Studies investigating the clinicopathological findings and prognosis of thyroid cancer in GD patients have reported inconsistent data. Some have reported more aggressiveness [14] and higher recurrence risk in GD patients compared with euthyroid patients $[15,16]$. However, others have reported no differences in the clinical characteristics or outcomes of thyroid cancer arising in GD patients [17,18], and one study reported a better prognosis of thyroid cancer in GD patients [19]. These inconsistencies may be related to the small sample sizes and variety of clinical settings in previous studies. Therefore, the present retrospective multicenter study was conducted to evaluate the clinicopathological features and outcomes of thyroid cancer in GD patients in the era of widespread application of US.

\section{METHODS}

\section{Baseline characteristic of GD patients}

This retrospective multicenter study was conducted at three tertiary referral hospitals in South Korea: Asan Medical Center,
Chonnam National University Hwasun Hospital, and Pusan National University Hospital. Data were collected for all GD patients who underwent thyroid cancer surgery between January 2010 and December 2019. The GD diagnostic criteria were defined as biochemical evidence of hyperthyroidism with serum anti-thyrotropin receptor antibody (thyrotropin binding inhibiting immunoglobulin [TBII]), and/or increased diffuse ${ }^{123} \mathrm{I}$ or ${ }^{99} \mathrm{mTc}$-pertechnetate uptake on a radionuclide scan, and/or the presence of clinical features, such as goiter or Graves' ophthalmopathy [20]. Patients in whom US was performed at least once during follow-up of GD were included. Thyroid US was performed using iU22 unit (Philips Healthcare, Bothell, WA, USA), EUB-7500 unit (Hitachi Medical Systems, Tokyo, Japan), GE Logiq E8 and E9 (Milwaukee-Brookfield, WI, USA), or Siemens ACUSON S2000 (Malvern, PA, USA) with a linear high-frequency probe (5 to $14 \mathrm{MHz}$ ). Patients who were followed up for less than 1 year were excluded.

The final study population consisted of 262 GD patients with coexisting thyroid cancer among 15,159 GD patients during the study period. The study protocol was approved by the Institutional Review Board (IRB) of each participating institute and the need for informed consent was waived due to the retrospective design (IRB approval numbers; IRB 2020-1776, CNUHH2021-136, 2011-008-096).

\section{Assessment of Graves' disease}

Demographic data including age, sex, smoking status, and prevalence of comorbidities, including hypertension, diabetes mellitus, dyslipidemia, and cardiovascular disease were collected from medical records. A family history of GD, GD activity status, type of anti-thyroid medication, and the TBII titer at the time of GD diagnosis were investigated retrospectively. Active GD status was defined as newly diagnosed GD or taking anti-thyroid drugs. The reference range of TBII was 0 to $1.5 \mathrm{IU} / \mathrm{L}$. GD patients were divided into two groups according to the presence or absence of the thyroid nodules other than thyroid cancer (index nodules): nodular GD and non-nodular GD groups (Fig. 1).

\section{Assessment of thyroid cancer}

Medical records yielded clinicopathological findings including coexisting thyroid cancer, tumor size, histological type, presence of extra-thyroidal extension (ETE), presence of cervical lymph node (LN) metastasis, number and longest diameter of metastatic LN, presence of distant metastasis, and initial tumornode-metastasis (TNM) stage according to the 8th edition of the American Joint Committee on Cancer/Union for International 


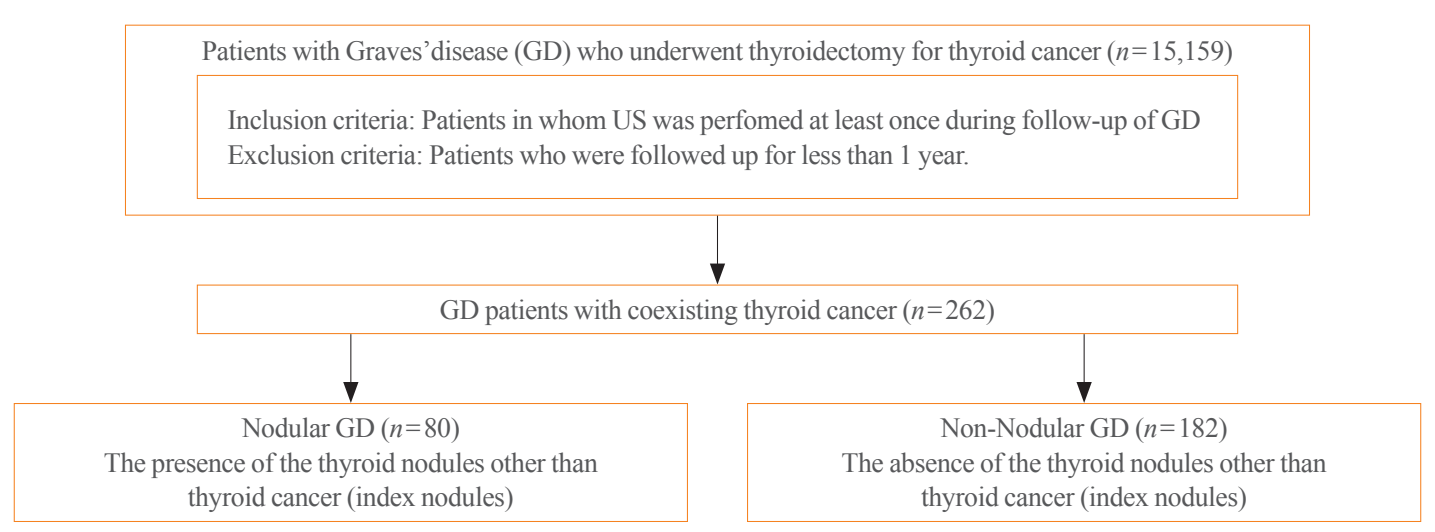

Fig. 1. Flow chart of the study population. US, ultrasonography.

Cancer Control TNM staging system [21], presence of the thyroid nodules other than thyroid cancer (index nodules), thyroid volume (g), extent of surgery, and use of radioactive iodine (RAI) therapy with total dose (mCi).

\section{Follow-up and clinical outcome assessments}

Serum TSH, free thyroxine, anti-thyroglobulin (Tg) antibody, and Tg levels were measured every 6 to 12 months during follow-up. Regular US follow-up was also performed every 6 to 12 months, and fine-needle aspiration (FNA) and/or washout fluid Tg measurements were conducted to detect recurrence if suspicious lesions were observed. Clinical outcomes of thyroid cancer were divided into remission, recurrence, persistence of biochemical disease, and persistence of structural disease as follows; remission, no clinical, biochemical, or structural evidence of disease; recurrence, evidence of disease after at least 1 year of remission; persistence of biochemical disease, persistently abnormal suppressed and/or stimulated Tg values or elevated anti$\mathrm{Tg}$ antibody titer without structural evidence of disease; and persistence of structural disease, structural or functional (RAI scan, ${ }^{18} \mathrm{~F}$-fluorodeoxyglucose positron emission tomography $\left.\left[{ }^{18} \mathrm{FDG}-\mathrm{PET}\right]\right)$ evidence of loco-regional or distant metastases.

\section{Statistical analysis}

Data are expressed as mean \pm standard deviation, median (interquartile range), or number (\%). Continuous variables were analyzed using Student's $t$ test, and categorical variables were analyzed using the chi-square test. Binary logistic regression analyses were used to assess the risk factors for recurrence/persistence of thyroid cancer. Continuous variable (TBII titer at diagnosis) with missing data were replaced with the mean value. All statistical analyses were performed using SPSS Statistics version 25 software (IBM Corp., Armonk, NY, USA), and a $P$ val- ue $<0.05$ was considered significant.

\section{RESULTS}

\section{Baseline characteristics of the GD patients}

Of the 15,159 GD patients treated during the study period, 262 $(1.7 \%)$ were diagnosed with thyroid cancer. Most patients underwent thyroid surgery due to cytologically suspicious or a proven malignancy $(n=251,95.8 \%)$ or other reasons, such as compressive symptoms due to a large goiter or relapsed and/or uncontrolled hyperthyroidism $(n=11,4.2 \%)$. Among the 262 patients, $245(93.5 \%)$ underwent total thyroidectomy and 17 (6.5\%) underwent lobectomy. Table 1 lists the baseline characteristics of these patients. The mean age was $55.1 \pm 13.9$ years, and $78.6 \%$ were female. Active GD status was observed in $87.4 \%$ ( $n=229)$ of the 262 patients; 200 patients had newly diagnosed GD, and 29 patients had recurrent GD. The mean TBII titer at the time of the first GD diagnosis was $16.0 \pm 19.6 \mathrm{IU} / \mathrm{L}$. Of the 262 patients, 11 (4.2\%) were diagnosed with occult thyroid cancer after total thyroidectomy for other reasons and 182 patients $(69.5 \%)$ had microcarcinoma. During the study period, 19,397 (64.2\%) of 30,213 non-GD patients underwent thyroid cancer surgery in three tertiary hospitals revealed to have microcarcinomas, which is comparable to GD patients.

\section{Clinicopathological and ultrasonographic findings of thyroid cancer according to the presence of nodules other than thyroid cancer in patients with GD}

The clinicopathological findings of GD patients with coexisting thyroid cancer were compared between the nodular GD and non-nodular GD groups (Table 2). The nodular GD group was older than the non-nodular group $(61.9 \pm 12.8$ years vs. $52.1 \pm$ 13.3 years, $P<0.001)$ and showed a female-dominance $(86.3 \%$ 
Table 1. Baseline Characteristics of Thyroid Cancer Patients with Graves' Disease $(n=262)$

\begin{tabular}{lc}
\hline Variable & $P$ value \\
\hline Age, yr & $55.1 \pm 13.9$ \\
Female sex & $206(78.6)$ \\
Disease status of GD & \\
Remission (past history) & $33(12.6)$ \\
Recurrence & $29(11.1)$ \\
The first diagnosis & $200(76.3)$ \\
Family history of GD & $15(5.7)$ \\
TBII at the time of the first diagnosis, IU/L & $16.0 \pm 19.6$ \\
Type of anti-thyroid medication & \\
Propylthiouracil & $29(11.1)$ \\
Methimazole & $208(79.4)$ \\
Carbimazole & $25(9.5)$ \\
Comorbidity & \\
Hypertension & $60(22.9)$ \\
Diabetes & $24(9.1)$ \\
Dyslipidemia & $44(16.8)$ \\
Cardiovascular disease & $20(7.6)$ \\
Smoking history & $251(95.8)$ \\
Non-smoker & $9(3.4)$ \\
Current smoker & $12(4.6)$ \\
Ex-smoker & \\
Detection of thyroid cancer & \\
Incidentaloma & \\
Per-operatively diagnosed by FNA & \\
\hline & \\
\hline
\end{tabular}

Values are expressed as mean \pm standard deviation or number (\%). GD, Graves' disease; TBII, thyrotropin binding inhibiting immunoglobulin; FNA, fine-needle aspiration.

vs. $75.3 \%, P=0.046)$. The GD activity and preoperative FNA results did not differ between the two groups. All patients in the non-nodular GD group had papillary thyroid carcinoma (PTC); the nodular GD group included two cases of non-PTC including one case of follicular carcinoma (FTC) and one case of poorly differentiated thyroid carcinoma (PDTC) $(P=0.043)$. Subgroup analysis of PTC revealed that more follicular variant PTC was observed in the nodular GD group than the non-nodular group. Seven cases subtypes of PTC other than follicular variant PTC were found only in the non-nodular GD group: three cases of tall cell variant PTC, one case of oncocytic variant PTC, two cases of solid variant PTC, and one case of diffuse sclerosing variant PTC (Table 3). Mean thyroid volume (g) was higher in the nodular GD group than in the non-nodular GD group
( $42.9 \pm 35.5$ g vs. $33.1 \pm 30.4 \mathrm{~g}, P=0.036)$. No significant differences in tumor size, bilaterality, lymphovascular invasion, ETE, or LN metastasis were observed between the two groups. Distant metastasis was not observed. RAI therapy was performed at similar rates in both groups $(42.5 \%$ vs. $43.4 \%, P=0.891)$. The clinical outcomes of thyroid cancer were similar between the two groups during the follow-up period (mean 59.1 months). At the last follow-up, 242 patients (92.4\%) were in thyroid cancer remission status. Recurrence was observed in eight patients (4.4\%) in the non-nodular GD group. Additionally, preoperative US findings of thyroid cancer did not differ between the nodular GD and non-nodular GD groups (Supplemental Table S1).

\section{Risk factors for recurrence/persistent thyroid cancer in patients with GD}

The univariate analysis revealed that tumor size (odds ratio [OR], 2.463; $P=0.001$ ), ETE (OR, 3.400; $P=0.010$ ), LN metastasis (OR, 9.260; $P<0.001$ ), and RAI therapy (OR, 8.618; $P=0$. $001)$ were significantly associated with the risk of recurrence/ persistent disease. However, the multivariate analysis, only LN metastasis (OR, 4.359; $P=0.020)$ was independently associated with recurrence/persistent disease (Table 4).

\section{DISCUSSION}

Unlike most previous studies investigating the prevalence of thyroid cancer in GD patients who underwent thyroidectomy, the present study enrolled all GD patients with thyroid cancer who were treated at three tertiary hospitals. Data from previous studies regarding the prevalence of coexisting thyroid cancer in GD patients assessed preoperatively by US are summarized in Supplemental Table S2 [9,16,22-30]. Studies using US to find thyroid nodules reported varying rates of concomitant thyroid cancer in GD patients were reported (3.3\% to $28.2 \%)$. The reported prevalence rates of coexisting thyroid cancers in GD patients have historically been lower, so some authors suggested that GD may have a protective effect against thyroid cancer, such as an anti-tumor effect via GD autoimmunity [6,31]; however, most thyroid cancers were detected as incidentalomas postoperatively or as palpable nodules preoperatively in the preUS era, which may have led the underestimation of thyroid cancer. To avoid selection and surveillance bias, many studies have compared the prevalence of occult papillary thyroid microcarcinoma (PTMC) in GD patients to those with multi-nodular goiters or chronic lymphocytic thyroiditis undergoing thyroidectomy for noncancer-related reasons [32,33]. These studies also 
Table 2. Clinicopathological Findings of Coexisting Thyroid Cancer between Nodular GD and Non-Nodular Graves' Disease ( $n=262)$

\begin{tabular}{|c|c|c|c|c|}
\hline Variable & Total & Nodular GD $(n=80)$ & Non-nodular GD $(n=182)$ & $P$ value \\
\hline Age, yr & $55.1 \pm 13.9$ & $61.9 \pm 12.8$ & $52.1 \pm 13.3$ & $<0.001$ \\
\hline Female sex & $206(78.6)$ & $69(86.3)$ & $137(75.3)$ & 0.046 \\
\hline Active GD & $229(87.4)$ & $70(87.5)$ & $159(87.4)$ & 0.975 \\
\hline Preoperative FNA results ${ }^{\mathrm{a}}$ & 251 & 74 & 177 & 0.351 \\
\hline Non-diagnostic & $18(7.2)$ & $6(8.1)$ & $12(6.6)$ & \\
\hline Benign & $3(1.2)$ & $1(1.4)$ & $2(1.2)$ & \\
\hline AUS/FLUS & $7(2.8)$ & $3(4.1)$ & $4(2.4)$ & \\
\hline Follicular neoplasm & $2(0.8)$ & $1(1.4)$ & $1(0.6)$ & \\
\hline Suspicious malignancy & $60(23.9)$ & $18(24.3)$ & $42(23.7)$ & \\
\hline Malignancy & $161(64.1)$ & $45(60.8)$ & $116(65.5)$ & \\
\hline Cancer type & & & & 0.043 \\
\hline PTC & $260(99.2)$ & $78(97.5)$ & $182(100)$ & \\
\hline FTC & $1(0.4)$ & $1(1.3)$ & 0 & \\
\hline PDTC & $1(0.4)$ & $1(1.3)$ & 0 & \\
\hline Type of operation & & & & 0.233 \\
\hline Lobectomy & $17(6.5)$ & $3(3.8)$ & $14(7.7)$ & \\
\hline Total thyroidectomy & $245(93.5)$ & $77(96.3)$ & $168(92.3)$ & \\
\hline \multicolumn{5}{|l|}{ Pathological findings } \\
\hline Tumor size, $\mathrm{cm}$ & $0.1 \pm 0.6$ & $0.9 \pm 0.7$ & $1.0 \pm 0.6$ & 0.358 \\
\hline Bilaterality & $54(20.6)$ & $20(25.0)$ & $34(18.7)$ & 0.244 \\
\hline Lymphovascular invasion & $26(10.0)$ & $7(8.8)$ & $19(10.5)$ & 0.664 \\
\hline ETE & & & & 0.653 \\
\hline No & $197(75.2)$ & $59(73.8)$ & $138(75.8)$ & \\
\hline Micro & $56(21.4)$ & $17(21.3)$ & $39(21.4)$ & \\
\hline Macro & $9(3.4)$ & $4(5.0)$ & $5(2.7)$ & \\
\hline LN metastasis & & & & 0.489 \\
\hline No & $173(66.0)$ & $56(70.0)$ & $117(64.3)$ & \\
\hline N1a & 73 (27.9) & $21(26.3)$ & $52(28.6)$ & \\
\hline $\mathrm{N} 1 \mathrm{~b}$ & $16(6.1)$ & $3(3.8)$ & $13(7.1)$ & \\
\hline Number of involved LNs & $1.8 \pm 6.0$ & $1.7 \pm 7.8$ & $1.9 \pm 5.0$ & 0.858 \\
\hline The largest diameter, $\mathrm{cm}$ & $0.5 \pm 0.6$ & $0.8 \pm 0.8$ & $0.5 \pm 0.6$ & 0.151 \\
\hline Thyroid gland volume, $\mathrm{g}^{\mathrm{b}}$ & $36.1 \pm 32.3$ & $42.9 \pm 35.5$ & $33.1 \pm 30.4$ & 0.036 \\
\hline RAI therapy & $113(43.1)$ & $34(42.5)$ & $79(43.4)$ & 0.891 \\
\hline Total dose of ${ }^{131} \mathrm{I}, \mathrm{mCi}$ & $99.6 \pm 75.7$ & $98.9 \pm 54.5$ & $99.9 \pm 83.8$ & 0.945 \\
\hline Clinical outcome & & & & 0.840 \\
\hline Remission & $242(92.4)$ & $76(95.0)$ & $166(91.2)$ & \\
\hline Recurrence & $8(3.1)$ & 0 & $8(4.4)$ & \\
\hline Persistent, structural & $9(3.4)$ & $2(2.5)$ & $7(3.8)$ & \\
\hline Persistent, biochemical & $3(1.1)$ & $2(2.5)$ & $1(0.5)$ & \\
\hline Mean follow-up, mo & $59.1 \pm 37.5$ & $57.8 \pm 36.7$ & $59.7 \pm 37.9$ & 0.713 \\
\hline
\end{tabular}

Values are expressed as mean \pm standard deviation or number $(\%)$.

GD, Graves' disease; FNA, fine needle aspiration; AUS, atypia of undetermined significance; FLUS, follicular lesion of undetermined significance; PTC, papillary thyroid carcinoma; FTC, follicular thyroid carcinoma; PDTC, poorly differentiated thyroid carcinoma; ETE, extrathyroidal extension; LN, lymph node; RAI, radioactive iodine.

${ }^{a}$ Data not available for 11 patients with occult thyroid cancer; ${ }^{b}$ Patients who underwent total thyroidectomy were included $(n=245)$. 
yielded inconsistent findings due to their small sample sizes. Recent studies in the era of widespread adoption of US showed a higher prevalence rate of thyroid cancer in GD patients compared to earlier studies. The immune tolerance of AITD prevents thyroid failure, which may increase the risk for thyroid cancer in GD patients [34,35] and binding of thyroid-stimulating antibody (TSAb) to the thyrotropin receptor stimulates tumor formation and angiogenesis by upregulating various growth factors $[8,36]$.

In the present study, thyroid cancer was detected in only $1.7 \%$ of all GD patients during the study period, which is much lower than in most previous reports [4]. However, a Japanese study reported only 166 cytologically diagnosed thyroid cancers among

Table 3. Subgroup Analysis of Cancer Subtype in Graves' Disease Patients with Coexisting Papillary Thyroid Cancer $(n=260)$

\begin{tabular}{lccc}
\hline Subtype & $\begin{array}{c}\text { Total } \\
(n=260)\end{array}$ & $\begin{array}{c}\text { Nodular GD } \\
(n=78)\end{array}$ & $\begin{array}{c}\text { Non-nodular } \\
\text { GD }(n=182)\end{array}$ \\
\hline Classic & $231(88.8)$ & $65(83.3)$ & $166(91.2)$ \\
Follicular variant & $22(8.5)$ & $13(16.7)$ & $9(4.9)$ \\
Tall cell variant & $3(1.2)$ & 0 & $3(1.6)$ \\
Oncocytic variant & $1(0.4)$ & 0 & $1(0.5)$ \\
Solid variant & $2(0.8)$ & 0 & $2(1.1)$ \\
Diffuse sclerosing & $1(0.4)$ & 0 & $1(0.5)$ \\
\hline \multicolumn{2}{l}{ Values are expressed as number $(\%)}$. & & \\
GD, Graves' disease. &
\end{tabular}

32,200 GD patients who were treated and underwent thyroid US between 1994 and 2004 in an outpatient clinic [22], which was an even lower incidence of thyroid cancer than in our study. A prospective Korean study reported a $3.3 \%$ prevalence of thyroid cancer in 245 GD patients after screening US was performed in all patients [23]. In only a few studies, US was performed in all GD patients, including non-surgically treated patients as in our study, and these studies reported lower incidences of thyroid cancer. A recent multicenter study performing preoperative US in all GD patients reported that only $38.3 \%$ of GD patients with coexisting thyroid cancer underwent thyroidectomy with suspicious FNA results and the ratio of microcarcinoma $(<1 \mathrm{~cm})$ was significantly higher in GD patients compared to age, sex, and tumor size-matched non-GD patients ( $60 \%$ vs. $37 \%$ ) [37]. Our study revealed a similar ratio of microcarcinoma in GD patients, even though thyroid cancer was diagnosed by preoperative US-guided FNA in $95.8 \%$ of GD patients. The increased ratio of PTMC in GD patients in recent studies could be related to the use of high-resolution US, which has been linked to an increase in thyroid cancer incidence in the last two decades [38]. In particular, a remarkable rise in thyroid cancer incidence has been observed in Korea [39].

A previous review and meta-analysis found that the presence of thyroid nodules increases the risk of thyroid cancer $[4,13]$. Up to half of GD patients with coexisting thyroid cancer have a nodular goiter on preoperative US [9], and we also found that 80 of the 262 GD patients had other thyroid nodule other than

Table 4. The Risk Factors for Recurrent/Persistent Disease of Thyroid Cancer in Patients with Graves' Disease

\begin{tabular}{|c|c|c|c|c|c|c|}
\hline \multirow{2}{*}{ Variable } & \multicolumn{3}{|c|}{ Univariate analysis } & \multicolumn{3}{|c|}{ Multivariate analysis } \\
\hline & OR & $95 \% \mathrm{CI}$ & $P$ value & OR & $95 \% \mathrm{CI}$ & $P$ value \\
\hline Age, yr & 0.980 & $0.949-1.013$ & 0.228 & & & \\
\hline Male sex & 0.472 & $0.179-1.245$ & 0.129 & & & \\
\hline TBII titer at diagnosis & 1.322 & $0.292-5.978$ & 0.717 & & & \\
\hline Activity of GD & 1.249 & $0.275-5.662$ & 0.773 & & & \\
\hline Tumor size & 2.463 & $1.454-4.174$ & 0.001 & 1.564 & $0.838-2.919$ & 0.160 \\
\hline Multifocality & 1.350 & $0.531-3.435$ & 0.529 & & & \\
\hline ETE & 3.400 & $1.346-8.589$ & 0.010 & 1.482 & $0.517-4.245$ & 0.464 \\
\hline Lymphovascular invasion & 0.455 & $0.058-3.543$ & 0.452 & & & \\
\hline LN metastasis & 9.260 & $2.993-28.653$ & $<0.001$ & 4.359 & $1.267-14.994$ & 0.020 \\
\hline Extent of surgery & 1.160 & $0.411-3.271$ & 0.779 & & & \\
\hline RAI therapy & 8.618 & $2.459-30.204$ & 0.001 & 3.138 & $0.782-12.593$ & 0.107 \\
\hline
\end{tabular}

Age, TBII titer at diagnosis, and tumor size are analyzed as continuous variables.

OR, odd ratio; CI, confidential interval; TBII, thyrotropin binding inhibiting immunoglobulin; GD, Graves' disease; ETE, extra-thyroidal extension; LN, lymph node; RAI, radioactive iodine. 
thyroid cancer. Our study analyzed clinical characteristics and prognosis of thyroid cancer arising from GD according to the presence of multi-nodularity to determine the association between thyroid autoimmune nodular changes and thyroid cancer. Nodular GD was more frequently observed in older women, but we found no difference in thyroid cancer characteristics at the time of the thyroid cancer diagnosis except for the histological type. FTC and PDTC were observed in only nodular GD patients, but almost all GD patients had PTC. All cases of the aggressive subtypes of PTC, such as the tall cell variant and solid variant, were observed in non-nodular GD patients. A study conducted in Greece [40] found a higher incidence of tall cell variant PTC in GD patients than non-GD patients, but we found a relatively low incidence of tall cell variant PTC in GD patients, which could be due to ethnic differences. Our subgroup analysis of US findings for thyroid cancer revealed no differences according to the presence of other thyroid nodules. It is important to screen for well-known suspicious US features [41] when attempting to detect thyroid cancer.

Previous studies investigating the link between GD and the prognosis of coexisting thyroid cancer have reported inconsistent data. One study found that in patients with thyroid cancers $<1 \mathrm{~cm}$, GD patients had an excellent prognosis and longer disease-free survival compared to age, sex, and tumor sizematched non-GD patients [19]. However, a meta-analysis found increased multifocality and distant metastasis at the time of the differentiated thyroid carcinoma (DTC) diagnosis in GD patients compared to non-GD patients [42]. A small study with a relatively long follow-up period (median 165.6 months) observed that GD patients with coexisting DTC had a poorer prognosis compared with age, sex, and tumor size-matched non-GD patients [43]. The same study found that 33.3\% (7/21) of GD patients and $10.0 \%(7 / 70)$ of non-GD patients were classified as stage IV on the TNM 7th edition [43]. It reported that six of the GD patients died, including three patients with distant metastasis at the time of the thyroid cancer diagnosis, whereas only one patient died in the non-GD group; However, it observed recurrent/persistent disease in $14.3 \%$ of the GD patients with DTC with only biochemical evidence, but in $10.0 \%$ of non-GD patients with structural evidence including LN and distant metastases [43]. In the present study, we did not detect any distant metastasis in any of the GD patients with DTC, which may have been due to early detection of thyroid cancer at the time of the GD diagnosis. We observed recurrence of thyroid cancer only in non-nodular GD patients, although the difference was not significant. GD may not harm the prognosis of non-aggressive thy- roid cancer, although this finding is based on nodular GD reported as chronic stimulation of TSAbs. Only LN metastasis was an independent prognostic factor for recurrence/persistent disease of thyroid cancer in GD patients. Advanced thyroid cancer with metastasis or a more aggressive histological type could be stimulated by the pathomechanism of GD, so early metastatic spread and increased mortality may occur.

This study had several limitations due to its retrospective design. We could not directly compare the prevalence of thyroid cancer between GD patients and non-GD patients. Additionally, the association between TBII in thyroid cancer and GD patients was evaluated using only the TBII titer at the time of the GD diagnosis. We could not evaluate the association with serial follow-up TBII and thyroid-stimulating immunoglobulin on thyroid cancer due to a lack of data. The follow-up period for thyroid cancer was also relatively short to evaluate the long-term clinical outcome. Large-scale prospective studies are necessary to accurately investigate the association between thyroid cancer and GD.

In conclusion, we found that the prevalence of thyroid cancer was much lower than in previous reports of GD patients. Additionally, more than $60 \%$ of the thyroid cancers arising from GD were microcarcinomas even though most of the thyroid cancers were diagnosed preoperatively by US-guided FNA. The clinical outcomes of thyroid cancer in GD patients were also excellent but, closer follow-up is necessary for patients with LN metastasis in the same way as for thyroid cancer in non-GD patients.

\section{CONFLICTS OF INTEREST}

No potential conflict of interest relevant to this article was reported.

\section{AUTHOR CONTRIBUTIONS}

Conception or design: W.B.K., Y.K.S., M.J.J., H.C.K. Acquisition, analysis, or interpretation of data: M.J., M.K., A.R.H., H.K.K., B.H.K. Drafting the work or revising: J.H.Y. Final approval of the manuscript: M.J.J., H.C.K.

\section{ORCID}

Jee Hee Yoon https://orcid.org/0000-0002-5919-6162

Min Ji Jeon https://orcid.org/0000-0002-1297-107X

Ho-Cheol Kang https://orcid.org/0000-0002-0448-1345 


\section{REFERENCES}

1. Smith TJ, Hegedus L. Graves' disease. N Engl J Med 2016; 375:1552-65.

2. Wemeau JL, Klein M, Sadoul JL, Briet C, Velayoudom-Cephise FL. Graves' disease: introduction, epidemiology, endogenous and environmental pathogenic factors. Ann Endocrinol (Paris) 2018;79:599-607.

3. Bartalena L. Diagnosis and management of Graves disease: a global overview. Nat Rev Endocrinol 2013;9:724-34.

4. Staniforth JU, Erdirimanne S, Eslick GD. Thyroid carcinoma in Graves' disease: a meta-analysis. Int J Surg 2016;27: 118-25.

5. Gabriele R, Letizia C, Borghese M, De Toma G, Celi M, Izzo L, et al. Thyroid cancer in patients with hyperthyroidism. Horm Res 2003;60:79-83.

6. Sokal JE. Incidence of malignancy in toxic and nontoxic nodular goiter. J Am Med Assoc 1954;154:1321-5.

7. Rieger R, Pimpl W, Money S, Rettenbacher L, Galvan G. Hyperthyroidism and concurrent thyroid malignancies. Surgery 1989;106:6-10.

8. Pazaitou-Panayiotou K, Michalakis K, Paschke R. Thyroid cancer in patients with hyperthyroidism. Horm Metab Res 2012;44:255-62.

9. Keskin C, Sahin M, Hasanov R, Aydogan BI, Demir O, Emral R, et al. Frequency of thyroid nodules and thyroid cancer in thyroidectomized patients with Graves' disease. Arch Med Sci 2019;16:302-7.

10. Shu X, Ji J, Li X, Sundquist J, Sundquist K, Hemminki K. Cancer risk in patients hospitalised for Graves' disease: a population-based cohort study in Sweden. Br J Cancer 2010; 102:1397-9.

11. Chen YK, Lin CL, Chang YJ, Cheng FT, Peng CL, Sung FC, et al. Cancer risk in patients with Graves' disease: a nationwide cohort study. Thyroid 2013;23:879-84.

12. Belfiore A, Russo D, Vigneri R, Filetti S. Graves' disease, thyroid nodules and thyroid cancer. Clin Endocrinol (Oxf) 2001;55:711-8.

13. Papanastasiou A, Sapalidis K, Goulis DG, Michalopoulos N, Mareti E, Mantalovas S, et al. Thyroid nodules as a risk factor for thyroid cancer in patients with Graves' disease: a systematic review and meta-analysis of observational studies in surgically treated patients. Clin Endocrinol (Oxf) 2019;91: $571-7$

14. Ozaki O, Ito K, Kobayashi K, Toshima K, Iwasaki H, Yashiro T. Thyroid carcinoma in Graves' disease. World J Surg
1990;14:437-41.

15. Menon R, Nair CG, Babu M, Jacob P, Krishna GP. The outcome of papillary thyroid cancer associated with Graves' disease: a case control study. J Thyroid Res 2018;2018:8253094.

16. Cappelli C, Braga M, De Martino E, Castellano M, Gandossi E, Agosti B, et al. Outcome of patients surgically treated for various forms of hyperthyroidism with differentiated thyroid cancer: experience at an endocrine center in Italy. Surg Today 2006;36:125-30.

17. Kasuga Y, Sugenoya A, Kobayashi S, Masuda H, Iida F. The outcome of patients with thyroid carcinoma and Graves' disease. Surg Today 1993;23:9-12.

18. Hales IB, McElduff A, Crummer P, Clifton-Bligh P, Delbridge L, Hoschl R, et al. Does Graves' disease or thyrotoxicosis affect the prognosis of thyroid cancer. J Clin Endocrinol Metab 1992;75:886-9.

19. Kikuchi S, Noguchi S, Yamashita H, Uchino S, Kawamoto H. Prognosis of small thyroid cancer in patients with Graves' disease. Br J Surg 2006;93:434-9.

20. Ross DS, Burch HB, Cooper DS, Greenlee MC, Laurberg P, Maia AL, et al. 2016 American Thyroid Association guidelines for diagnosis and management of hyperthyroidism and other causes of thyrotoxicosis. Thyroid 2016;26:1343-421.

21. Amin MB, Edge SB, Greene FL, Byrd DR, Brookland RK, Washington MK, et al. 2017 AJCC Cancer Staging Manual. 8th ed. New York: Springer; 2017.

22. Yano Y, Shibuya H, Kitagawa W, Nagahama M, Sugino K, Ito K, et al. Recent outcome of Graves' disease patients with papillary thyroid cancer. Eur J Endocrinol 2007;157:325-9.

23. Kim WB, Han SM, Kim TY, Nam-Goong IS, Gong G, Lee $\mathrm{HK}$, et al. Ultrasonographic screening for detection of thyroid cancer in patients with Graves' disease. Clin Endocrinol (Oxf) 2004;60:719-25.

24. Kraimps JL, Bouin-Pineau MH, Mathonnet M, De Calan L, Ronceray J, Visset J, et al. Multicentre study of thyroid nodules in patients with Graves' disease. Br J Surg 2000;87:11113.

25. Erbil Y, Barbaros U, Ozbey N, Kapran Y, Tukenmez M, Bozbora A, et al. Graves' disease, with and without nodules, and the risk of thyroid carcinoma. J Laryngol Otol 2008; 122:291-5.

26. Ren M, Wu MC, Shang CZ, Wang XY, Zhang JL, Cheng H, et al. Predictive factors of thyroid cancer in patients with Graves' disease. World J Surg 2014;38:80-7.

27. Ergin AB, Saralaya S, Olansky L. Incidental papillary thyroid carcinoma: clinical characteristics and prognostic fac- 
tors among patients with Graves' disease and euthyroid goiter, Cleveland Clinic experience. Am J Otolaryngol 2014;35: 784-90.

28. Tam AA, Kaya C, Kilic FB, Ersoy R, Cakir B. Thyroid nodules and thyroid cancer in Graves' disease. Arq Bras Endocrinol Metabol 2014;58:933-8.

29. Alvarez AL, Mulder M, Handelsman RS, Lew JI, Farra JC. High rates of underlying thyroid cancer in patients undergoing thyroidectomy for hyperthyroidism. J Surg Res 2020; 245:523-8.

30. Casella C, Morandi R, Verrengia A, Galani A, Molfino S, Cuka D, et al. Thyroid cancer and nodules in Graves' disease: a single center experience. Endocr Metab Immune Disord Drug Targets 2021;21:2028-34.

31. Imam S, Dar P, Paparodis R, Almotah K, Al-Khudhair A, Hasan SA, et al. Nature of coexisting thyroid autoimmune disease determines success or failure of tumor immunity in thyroid cancer. J Immunother Cancer 2019;7:3.

32. Paparodis RD, Karvounis E, Bantouna D, Chourpiliadis C, Chourpiliadi H, Livadas S, et al. Incidentally discovered papillary thyroid microcarcinomas are more frequently found in patients with chronic lymphocytic thyroiditis than with multinodular goiter or Graves' disease. Thyroid 2020;30:531-5.

33. Jia Q, Li X, Liu Y, Li L, Kwong JS, Ren K, et al. Incidental thyroid carcinoma in surgery-treated hyperthyroid patients with Graves' disease: a systematic review and meta-analysis of cohort studies. Cancer Manag Res 2018;10:1201-7.

34. Lee HJ, Li CW, Hammerstad SS, Stefan M, Tomer Y. Immunogenetics of autoimmune thyroid diseases: a comprehensive review. J Autoimmun 2015;64:82-90.

35. Ferrari SM, Fallahi P, Elia G, Ragusa F, Ruffilli I, Paparo $\mathrm{SR}$, et al. Thyroid autoimmune disorders and cancer. Semin Cancer Biol 2020;64:135-46.

36. Filetti S, Belfiore A, Amir SM, Daniels GH, Ippolito O, Vigneri $\mathrm{R}$, et al. The role of thyroid-stimulating antibodies of
Graves' disease in differentiated thyroid cancer. N Engl J Med 1988;318:753-9.

37. Premoli P, Tanda ML, Piantanida E, Veronesi G, Gallo D, Masiello E, et al. Features and outcome of differentiated thyroid carcinoma associated with Graves' disease: results of a large, retrospective, multicenter study. J Endocrinol Invest 2020;43:109-16.

38. Vaccarella S, Franceschi S, Bray F, Wild CP, Plummer M, Dal Maso L. Worldwide thyroid-cancer epidemic?: the increasing impact of overdiagnosis. N Engl J Med 2016;375: 614-7.

39. Vaccarella S, Dal Maso L, Laversanne M, Bray F, Plummer M, Franceschi S. The impact of diagnostic changes on the rise in thyroid cancer incidence: a population-based study in selected high-resource countries. Thyroid 2015;25:1127-36.

40. Boutzios G, Vasileiadis I, Zapanti E, Charitoudis G, Karakostas E, Ieromonachou P, et al. Higher incidence of tall cell variant of papillary thyroid carcinoma in Graves' disease. Thyroid 2014;24:347-54.

41. Haugen BR, Alexander EK, Bible KC, Doherty GM, Mandel SJ, Nikiforov YE, et al. 2015 American Thyroid Association management guidelines for adult patients with thyroid nodules and differentiated thyroid cancer: the American Thyroid Association guidelines task force on thyroid nodules and differentiated thyroid cancer. Thyroid 2016;26:1133.

42. Mekraksakit P, Rattanawong P, Karnchanasorn R, Kanitsoraphan C, Leelaviwat N, Poonsombudlert K, et al. Prognosis of differentiated thyroid carcinoma in patients with Graves disease: a systematic review and meta-analysis. Endocr Pract 2019;25:1323-37.

43. Pellegriti G, Mannarino C, Russo M, Terranova R, Marturano I, Vigneri R, et al. Increased mortality in patients with differentiated thyroid cancer associated with Graves' disease. J Clin Endocrinol Metab 2013;98:1014-21. 